\title{
Association between age at menarche and risk of diabetes in adults: results from the EPIC-Norfolk cohort study
}

\author{
R. Lakshman • N. Forouhi • R. Luben • S. Bingham • \\ K. Khaw $\cdot$ N. Wareham $\cdot$ K. K. Ong
}

Received: 7 December 2007 / Accepted: 18 January 2008/Published online: 5 March 2008

(C) Springer-Verlag 2008

\begin{abstract}
Aims/hypothesis Earlier age at menarche is associated with increased BMI and obesity risk from early childhood through to adulthood. We hypothesised that earlier age at menarche would also predict subsequent diabetes risk.

Methods This was a population-based prospective cohort study of 13,308 women, who were aged 40 to 75 years between 1993 and 1997 and participating in the Norfolk cohort of the European Prospective Investigation into Cancer and Nutrition (EPIC-Norfolk). We used data on age at menarche and ascertained diabetes incidence to 2005 .

Results There were 734 cases of diabetes (363 incident and 371 prevalent cases). Mean age at menarche was lower in women with diabetes than in non-diabetic women (12.8 vs 13.0 years, $p=0.008)$. Compared with the earliest quintile (menarche at 8-11 years), women in the oldest quintile (menarche at $15-18$ years) had lower BMI ( $25.5 \mathrm{vs} 27.4 \mathrm{~kg} / \mathrm{m}^{2}$, $p<0.0001)$ and a reduced risk of diabetes (OR $0.66[95 \% \mathrm{CI}$
\end{abstract}

R. Lakshman $\cdot$ N. Forouhi $\cdot$ N. Wareham $\cdot$ K. K. Ong $(\bowtie)$

MRC Epidemiology Unit,

Institute of Metabolic Science, Addenbrooke's Hospital,

Box 285, Cambridge CB2 0QQ, UK

e-mail: ken.ong@mrc-epid.cam.ac.uk

R. Luben $\cdot$ K. Khaw

Department of Public Health and Primary Care,

Institute of Public Health, University of Cambridge,

Cambridge, UK

S. Bingham

Centre for Nutritional Epidemiology in Cancer Prevention and

Survival, University of Cambridge,

Cambridge, UK

K. K. Ong

Department of Paediatrics, University of Cambridge,

Cambridge, UK
0.51-0.86] adjusted for age, family history, physical activity, smoking, occupational social class, parity and use of hormonal preparations). The association between age at menarche and diabetes was linear (adjusted OR 0.91 [95\% CI 0.87-0.96] per 1 year later menarche) and appeared to be completely mediated by adult BMI or waist circumference (OR 0.98 [95\% CI $0.93-$ 1.03], further adjusted for BMI at age 40-75 years).

Conclusions/interpretation Earlier age at menarche increases the risk of diabetes in women and this association appears to be mediated by increased adiposity. History of earlier menarche may help to identify women with increased subsequent risk of diabetes.

Keywords Body mass index $\cdot$ Diabetes $\cdot$ EPIC $\cdot$ Menarche Waist circumference $\cdot$ Women

\section{Abbreviation \\ EPIC European Prospective Investigation into Cancer and Nutrition}

\section{Introduction}

With the increasing prevalence of type 2 diabetes worldwide $[1,2]$, identification of early risk factors that predispose to type 2 diabetes has become a priority. Obesity is a wellrecognised risk factor for impaired glucose tolerance and diabetes [3, 4] that appears to track from childhood [5]. Overweight and obese girls have earlier onset of puberty and younger age at menarche (onset of menstruation) [6-9]. These individuals with earlier menarche retain markedly higher risks for obesity throughout adult life. For instance in the Avon Longitudinal Study of Parents and Children (ALSPAC), mothers showed a fivefold difference in pre- 
pregnancy obesity risk across the quintiles of age at menarche [10].

Previous studies have reported associations between earlier age at menarche and higher blood glucose levels in adults, but they may have been underpowered to detect any association with diabetes risk [11-13]. We hypothesised that earlier age at menarche would also predict subsequent diabetes risk. We therefore studied the association between age at menarche and risk of diabetes in later life in a large population-based study, the Norfolk cohort of the European Prospective Investigation into Cancer and Nutrition (EPIC).

\section{Methods}

Study design and population The EPIC-Norfolk cohort is part of a multi-centre international study designed to investigate the relationship between diet, cancer and chronic disease. The study design has been previously described [14]. Briefly, in Norfolk UK, 25,639 volunteers aged 40 to 75 years were recruited between 1993 and 1997. Participants attended for a baseline health check (19931997) and had three follow-up assessments (postal questionnaire at 18 months, a second health check between 1998 and 2000, and postal questionnaire between 2002 and 2005). A detailed health and lifestyle questionnaire was completed at each stage.

At baseline, questionnaire data were collected on family history of diabetes (yes/no), number of siblings, smoking status (current, former or never), occupational social class (professional, managerial and technical, skilled non-manual, skilled manual, partly skilled manual, unskilled) and highest educational qualification (none, O-level or equivalent, A-level or equivalent, degree level). A four-point physical activity index incorporating occupational and non-occupational physical activity was used to categorise individuals as sedentary, moderately inactive, active or very active [15]. Oral contraceptive use (current, former or never), hormone replacement therapy use (current, former or never) and parity (number of pregnancies) were also assessed by questionnaire at baseline. At the baseline health check, height, weight and both waist and hip circumferences were measured according to a standard protocol and blood samples were taken by the study nurses.

The study was approved by the local research ethics committee and participants gave written informed consent.

Ascertainment of age at menarche and diabetes Age at menarche in completed whole years was ascertained by recall in the baseline questionnaire. Cases of diabetes, both prevalent and incident, were clinically ascertained. Cases of diabetes prevalent at baseline were identified by: self-report of physician diagnosis, diabetes medication reported or brought into first health check visit or report of being on a diabetes diet in the health and lifestyle questionnaire. Clinically incident cases of diabetes were ascertained using multiple sources of information among participants who did not have prevalent diabetes. Sources internal to the study included any self-report of diabetes diagnosed by a doctor and of diabetes-specific medication at follow-up. Record linkage with external sources ascertained clinically incident diabetes from: general practice diabetes registers, the local hospital diabetes register, hospital admissions data for diabetes-related admissions and Office for National Statistics mortality data with coding for diabetes. Identification of diabetes cases through external sources was performed independently of the health check and questionnaire followup. Possible incident cases based solely on self-report and not confirmed by another data source did not qualify as confirmed cases. Cases ascertained up to December 2005 were included in this study.

Statistical analysis Analyses were restricted to the 13,308 women who reported their menarche at a physiological age, which has previously been defined for the EPIC cohort as between 8 and 18 years [16].

We used the following three approaches to studying the association between the exposure variable (age at menarche) and diabetes (outcome variable): (1) as a binary variable, i.e. odds of diabetes for women above and below the median age at menarche (13.0 years in current study); (2) as a linear association, i.e. OR per year delay in onset of menarche; and (3) based on quintiles for age at menarche (8-11, 12, 13, 14 and 15-18 years).

We performed multivariate logistic regression analyses to adjust for a range of possible confounding and mediating factors. Since there was a significant positive correlation between age at menarche and age at baseline assessment that reflected the recognised birth cohort effect [16], all analyses included adjustment for age at baseline assessment. Subsequent models were built with staged inclusions of: possible confounding factors (physical activity, family history of diabetes, occupational social class, educational level, smoking); reproductive factors (parity, oral contraceptive use and hormone replacement therapy); and potential mediating factors (BMI and waist circumference measured at age 40 to 75 years). Tests for interaction with BMI and waist circumference group were performed using the likelihood ratio test. Sensitivity analyses were performed to exclude the effect of potential cases with type1 diabetes.

All analyses were performed using STATA statistical software, version 9.2 (STATACORP, College Station, TX, USA). 


\section{Results}

Cohort characteristics In the whole cohort $(n=13,308$ women) there were 734 cases of diabetes (371 prevalent cases at baseline and 363 incident cases). The cumulative prevalence of diabetes at age 40 to 75 years was $2.8 \%$. Since the exposure (age at menarche) predated the outcome (onset of diabetes), we combined incident and prevalent cases.

Age at menarche showed a normal distribution (mean 13.0, median 13, SD 1.6 years) and was lower in women with diabetes than in non-diabetic women (12.8 vs 13.0 years, $p=0.008)$. Table 1 shows the characteristics of women by quintiles of age at menarche.

Association between age at menarche and diabetes Women with menarche at 13 years or later ( $\geq$ median) had a $27 \%$ lower risk of diabetes than those with menarche $<3$ years (OR 0.73 [95\% CI 0.63-0.86], $p<0.001$, adjusted for potential confounders). In a linear model, each 1 year delay in onset of menarche was associated with a $10 \%$ lower risk of diabetes (OR 0.90 [95\% CI 0.86-0.95], $p<0.001$, adjusted for age) and was robust to adjustment for potential confounders (Table 2). Examination of diabetes risk by quintiles of age at menarche confirmed a linear trend (likelihood ratio test for non-linearity: $p=0.3$ ). Compared with the earliest quintile (menarche at $8-11$ years), women in the oldest quintile (menarche at $15-18$ years) had a $34 \%$ lower risk of diabetes (OR 0.66 [95\% CI $0.51-0.85]$ adjusted for potential confounding factors; Fig. 1).

Self-reported birthweight was available for only 7,737 women. Further adjustment for birthweight did not materially alter the association between menarche and diabetes risk (OR 0.89 [95\% CI 0.83-0.95], $p<0.001$ ). Similarly, further adjustment for number of siblings did not materially alter the association (OR 0.91 [95\% CI $0.87-0.95], p<0.001$ ).

Mediation of the association by adiposity For each 1 year later onset of menarche, age-adjusted adult BMI was lower by $0.43 \mathrm{~kg} / \mathrm{m}^{2}$ per year $(95 \%$ CI $0.48-0.38, p<0.001)$ and waist circumference was lower by $0.74 \mathrm{~cm}$ per year $(95 \%$ CI $0.85-0.62, p<0.001)$. Mean BMI and waist circumference values and percentages of women who were obese at

Table 1 Characteristics of 13,308 women at age 40 to 75 years by quintiles of age at menarche: the EPIC-Norfolk cohort study

\begin{tabular}{|c|c|c|c|c|c|c|}
\hline \multicolumn{7}{|c|}{ Quintiles of age at menarche } \\
\hline $\begin{array}{l}\text { Variable } \\
n(\%)\end{array}$ & $\begin{array}{l}8-11 \text { years } \\
2,853(21)\end{array}$ & $\begin{array}{l}12 \text { years } \\
2,342(18)\end{array}$ & $\begin{array}{l}13 \text { years } \\
3,179(24)\end{array}$ & $\begin{array}{l}14 \text { years } \\
2,726(20)\end{array}$ & $\begin{array}{l}15-18 \text { years } \\
2,208(17)\end{array}$ & $p$ value $^{\mathrm{a}}$ \\
\hline $\begin{array}{l}\text { Age at baseline } \\
\text { (years) }\end{array}$ & $57.0(56.7-57.3)$ & $57.6(57.2-58.0)$ & $58.0(57.6-58.3)$ & $59.5(59.1-59.8)$ & $60.2(59.8-60.6)$ & $<0.001$ \\
\hline BMI $\left(\mathrm{kg} / \mathrm{m}^{2}\right)$ & $27.4(27.2-27.6)$ & $26.4(26.2-26.6)$ & $26.0(25.9-26.2)$ & $25.8(25.6-25.9)$ & $25.5(25.3-25.7)$ & $<0.001$ \\
\hline Waist (cm) & $83.9(83.5-84.3)$ & $82.3(81.9-82.8)$ & $81.6(81.3-82.0)$ & $81.4(81.0-81.8)$ & $81.3(80.9-81.7)$ & $<0.001$ \\
\hline Height (cm) & $160.3(160.1-160.6)$ & $161.0(160.7-161.2)$ & $161.1(160.9-161.3)$ & $161.2(160.9-161.4)$ & $161.3(161.1-161.6)$ & $<0.001$ \\
\hline Diabetes $^{\mathrm{b}}$ & $191(6.7)$ & $135(5.8)$ & $152(4.8)$ & $145(5.3)$ & $111(5.0)$ & $<0.001$ \\
\hline $\begin{array}{l}\text { Family history } \\
\text { of diabetes }\end{array}$ & $200(7.0)$ & $191(8.1)$ & $190(6.0)$ & $179(6.6)$ & $128(5.8)$ & 0.02 \\
\hline $\mathrm{BMI}>30 \mathrm{~kg} / \mathrm{m}^{2}$ & $695(24.4)$ & $415(17.8)$ & $508(16.0)$ & $362(13.3)$ & $284(12.9)$ & $<0.001$ \\
\hline Waist $>80 \mathrm{~cm}^{\mathrm{c}}$ & $1,645(57.7)$ & $1,203(51.4)$ & $1,561(49.1)$ & $1,338(49.1)$ & $1,079(48.9)$ & $<0.001$ \\
\hline Never smoked & $1,598(56.4)$ & $1,368(58.8)$ & $1,786(56.6)$ & $1,503(55.7)$ & $1,177(54.1)$ & 0.74 \\
\hline Never used HRT & $1,847(64.7)$ & $1,621(69.2)$ & $2,184(68.7)$ & $1,860(68.2)$ & $1,546(70.0)$ & 0.38 \\
\hline Never used OC & $1,447(50.7)$ & $1,202(51.3)$ & $1617(50.9)$ & $1,548(56.8)$ & $1,306(59.1)$ & 0.16 \\
\hline Low $\mathrm{PA}^{\mathrm{d}}$ & $799(28.0)$ & $672(28.7)$ & $925(29.1)$ & $874(32.1)$ & $742(33.6)$ & 0.23 \\
\hline $\begin{array}{l}\text { Non-manual } \\
\text { social class }\end{array}$ & $1,701(60.6)$ & $1,435(62.5)$ & $1,942(62.1)$ & $1,637(61.4)$ & $1,278(59.5)$ & 0.37 \\
\hline $\begin{array}{l}\text { A-level or } \\
\text { degree }^{f}\end{array}$ & $1,123(39.4)$ & $930(39.7)$ & $1,209(38.0)$ & $913(33.5)$ & 719 (32.6) & $<0.001$ \\
\hline
\end{tabular}

Values are unadjusted means $(95 \% \mathrm{CI})$ or $n(\%)$

HRT, hormone replacement therapy; OC, oral contraceptive

${ }^{a} p$ value for trend was calculated from regression analyses adjusted for age at baseline (except for analyses for age at baseline)

${ }^{\mathrm{b}}$ Diabetes status includes prevalent cases 1993-1997 and incident cases up to 2005

${ }^{\mathrm{c}}$ Waist $>80 \mathrm{~cm}$, waist circumference greater than $80 \mathrm{~cm}$

${ }^{\mathrm{d}}$ Low PA, lowest level of physical activity on a four-point scale

${ }^{\text {e }}$ Non-manual social class, professional, managerial and technical, skilled non-manual

${ }^{\mathrm{f}}$ Highest educational level 
Table 2 Logistic regression analyses of the association between age at menarche and the risk of diabetes: the EPIC-Norfolk cohort study

\begin{tabular}{|c|c|c|c|c|c|c|}
\hline & \multicolumn{2}{|l|}{ All diabetes cases } & \multicolumn{2}{|c|}{ Prevalent cases only } & \multicolumn{2}{|c|}{ Incident cases only } \\
\hline & OR $(95 \% \mathrm{CI})^{\mathrm{a}}$ & $p$ value & OR & $p$ value & OR & $p$ value \\
\hline Model $1^{\mathrm{b}}$ & $0.90(0.86-0.95)$ & $<0.001$ & 0.90 & 0.002 & 0.93 & 0.02 \\
\hline Model $2^{\mathrm{c}}$ & $0.91(0.87-0.96)$ & $<0.001$ & 0.90 & 0.004 & 0.92 & 0.007 \\
\hline Model $3^{\mathrm{d}}$ & $0.91(0.87-0.96)$ & $<0.001$ & 0.90 & 0.004 & 0.92 & 0.006 \\
\hline Model 4 (model 3+BMI) & $0.98(0.93-1.03)$ & 0.4 & 0.95 & 0.1 & 0.98 & 0.7 \\
\hline Model 5 (model 3+waist circumference) & $0.96(0.92-1.01)$ & 0.1 & 0.94 & 0.1 & 0.97 & 0.4 \\
\hline
\end{tabular}

${ }^{\text {a }}$ ORs are per 1 year delay in age at menarche

${ }^{\mathrm{b}}$ Adjusted for age at baseline assessment

${ }^{c}$ Model $1+$ confounding factors: smoking, occupational social class, educational level, physical activity, family history of diabetes

${ }^{\mathrm{d}}$ Model $2+$ reproductive factors parity, oral contraceptive use, hormone replacement therapy use

age 40 to 75 years by quintile of age at menarche confirm that the associations are linear (Table 1). For each 1 year delay in age at menarche, risk of obesity (BMI $>30 \mathrm{~kg} / \mathrm{m}^{2}$ ) at age 40 to 75 years was reduced by $\sim 20 \%$ (OR 0.83 [95\% CI 0.81-0.85] adjusted for age). Figure 2 shows that mean age at menarche was lower in women with increasing categories of overweight and obesity.

The association between age at menarche and diabetes was completely attenuated following adjustment for BMI or waist circumference at age 40 to 75 years (OR per 1 year later menarche onset 0.98 [95\% CI 0.93-1.03], $p=0.4$ adjusted for BMI; Table 2). The association between age at menarche and diabetes was also attenuated by excluding women with BMI $\geq 30 \mathrm{~kg} / \mathrm{m}^{2} \quad(n=2,281$; OR 0.95 [95\% CI $0.90-1.0], p=0.09$ ).

There was no interaction between BMI status and age at menarche on the risk of diabetes. However, the association between age at menarche and diabetes risk appeared to be more apparent in women with larger waist circumference

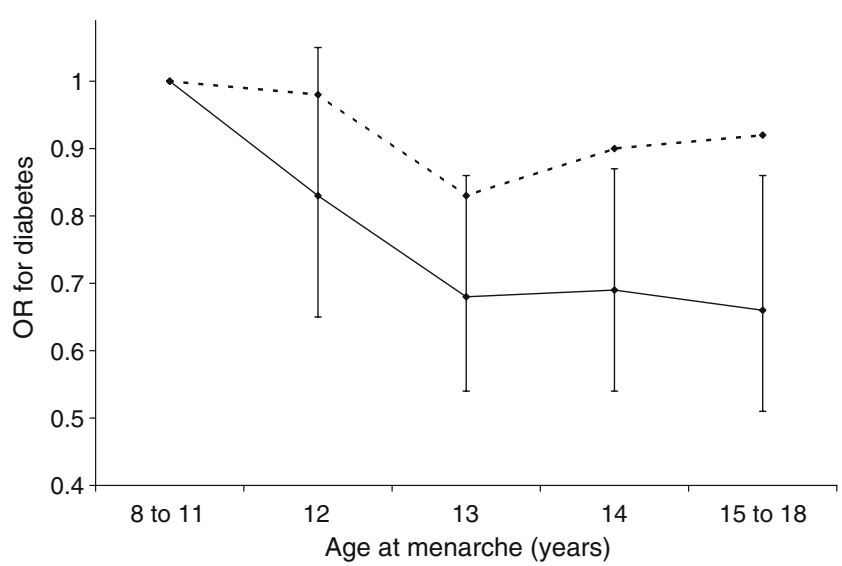

Fig. 1 ORs for diabetes by quintiles of age at menarche adjusted for potential confounding factors (solid line; $95 \%$ CIs shown by error bars; $p=0.001$ for trend), and further adjusted for BMI at age 40-75 years (dashed line; $p=0.59$ for trend): the EPIC-Norfolk cohort study
$(>80 \mathrm{~cm})$ than in women with smaller waist circumference (Table $3 ; p=0.03$ for interaction).

Sensitivity analyses Sensitivity analyses were performed to exclude the effect of potential cases with type 1 diabetes. Excluding cases with onset of diabetes before age 20 years $(n=29)$ had little impact on the association between age at menarche and diabetes risk (OR per 1 year later onset of menarche 0.91 [95\% CI 0.86-0.95], $p<0.001$ adjusted for potential confounding factors). The association, moreover, was very similar both in incident cases and in prevalent cases (Table 2). In this cohort, incident cases of diabetes were defined as women who (1) were non-diabetic at baseline examination and (2) developed diabetes during follow-up after age 40 years, making it very likely that they had type 2 diabetes.

\section{Discussion}

To our knowledge, this is the first study to demonstrate an association between earlier menarche and increased risk of diabetes in adults. The current study, of 734 diabetes patients from 13,308 mainly white European women is by far the largest to date. Compared with the earliest quintile of menarche onset, women in the oldest quintile had a $34 \%$ lower risk of diabetes.

Earlier studies have reported supportive evidence with glucose levels, but they were probably underpowered to detect an association with diabetes. The Rancho Bernardo Study [11] studied 997 women aged 50 to 92 years and reported that earlier menarche was associated with higher fasting and post-challenge plasma glucose levels. In a study of 121 women with polycystic ovary syndrome, Gambineri et al. [13] reported that earlier menarche onset was associated with impaired glucose tolerance. However, Cooper et al. [12] found no association between age at 


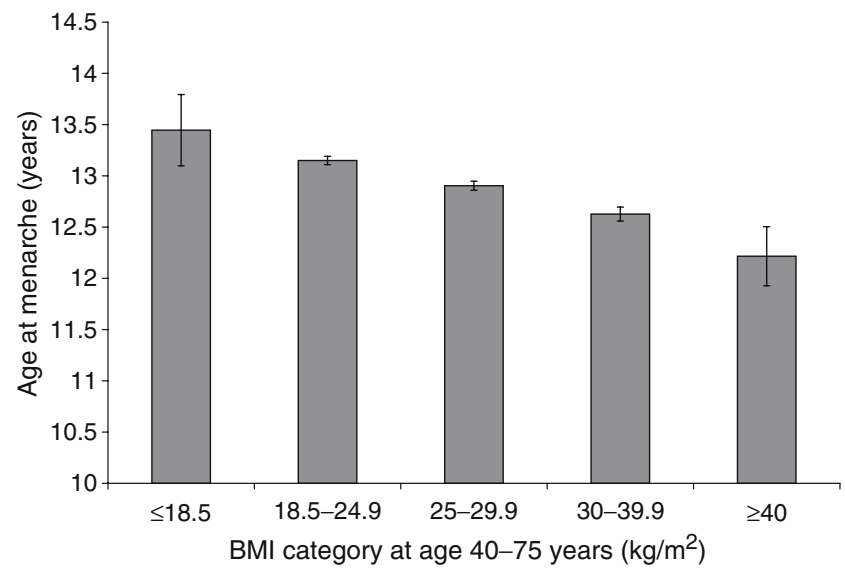

Fig. 2 Mean (95\% CIs) age at menarche by adult BMI category: the EPIC-Norfolk cohort study. $p<0.0001$ for trend

menarche and adult-onset diabetes in a prospective study of 668 white, college-educated women who had completed menstrual diaries throughout their reproductive years.

A limitation of our study was that age at menarche was reported by recall many years later. However, other studies have shown high correlations ( $r=0.67$ to 0.79 ) between age at menarche by recall during middle-age and the original childhood data $[17,18]$. Bias is unlikely and the observed association may therefore be slightly weakened by regression dilution. A further limitation was the lack of clinical distinction between type 1 and type 2 diabetes. Type 1 diabetes has been associated with delayed menarche [19], so any effect of including participants with type 1 diabetes would have attenuated the association between earlier menarche and type 2 diabetes. However, sensitivity analyses showed very robust associations after excluding juvenile-onset diabetes or after restriction to incident cases (i.e. those arising after age 40-75 years and therefore unlikely to be type 1 diabetes). Our data therefore strongly suggest that earlier age at menarche is associated with increased risk of type 2 diabetes. We did not have biochemical confirmation of diabetes or of normal glucose

Table 3 ORs for diabetes per 1 year increase in age at menarche for different strata of BMI and waist circumference: the EPIC-Norfolk cohort study

\begin{tabular}{lrll}
\hline & \multicolumn{1}{c}{$n$} & OR $(95 \% \mathrm{CI})^{\text {a }}$ & $p$ value \\
\hline $\mathrm{BMI} \geq 30 \mathrm{~kg} / \mathrm{m}^{2}$ & 2,281 & $0.94(0.87-1.01)$ & 0.10 \\
$\mathrm{BMI}<30 \mathrm{~kg} / \mathrm{m}^{2}$ & 10,978 & $0.95(0.90-1.00)$ & 0.09 \\
$\quad \begin{array}{l}\text { Waist circumference } \\
\quad \geq 80 \mathrm{~cm}\end{array}$ & 7,018 & $0.92(0.88-0.97)$ & 0.002 \\
$\begin{array}{l}\text { Waist circumference } \\
\quad<80 \mathrm{~cm}\end{array}$ & 6,241 & $0.98(0.88-1.09)$ & 0.72 \\
& & &
\end{tabular}

${ }^{\text {a }}$ Adjusted for age at baseline tolerance, so it is possible that unrecognised cases of undiagnosed type 2 diabetes could have weakened our observations.

The strengths of our study also merit consideration. This was a large population-based cohort study and anthropometry was performed by trained personnel at a health check visit. A comprehensive range of possible confounding and mediating factors, including physical activity and reproductive factors, were also measured and considered. Diabetes status was not dependent solely on self-report, but was ascertained and validated using several internal and external sources.

The association between earlier menarche and increased diabetes risk was completely explained by larger BMI or waist circumference at $40-75$ years. Further observation that this association was stronger in women with larger baseline waist circumference suggests that later life interventions to reduce central obesity may abolish the elevated risk of diabetes in women with earlier menarche. Previous large population studies have also reported a strong association between earlier menarche and larger adult BMI $[10,20]$. However, the causal direction between earlier menarche and BMI is much debated, as girls with early puberty onset already have higher than average BMI at that time $[21,22]$ and childhood insulin resistance may drive both the earlier onset and rapid progression through puberty [23]. Unfortunately we did not have data on childhood growth in this cohort. There is growing evidence from other large longitudinal studies that increased adiposity precedes the onset of puberty [8, 24, 25], while the rapid weight gain associated with earlier puberty may occur particularly during infancy $[10,26,27]$. The current finding, an association between earlier menarche and adult diabetes that is attenuated by adult BMI, might therefore be indicative of a risk pathway beginning with rapid infant weight gain and continuing with childhood-onset of overweight and obesity, and adult obesity. Alternatively, earlier rate of pubertal development might itself lead to differences in adiposity during adolescence and adulthood.

Earlier age at menarche has also been associated with several reproductive and cardiovascular-related disease outcomes, such as breast cancer [28-31] and ischaemic heart disease [32]. In Europe, age at menarche has decreased steadily since the nineteenth century by up to 12 months per decade [33]. However, over the past 50 years, the secular trend has probably slowed or even stopped in some populations [33], and it is unclear whether the secular trend in menarche age coincides with increasing rates of type 2 diabetes.

In conclusion, our study of a large population-based cohort provides convincing evidence that earlier age of menarche onset increases the risk of adult diabetes and this appears to be mediated by greater adult adiposity. By ascertaining a history of earlier menarche onset, it may be possible to identify women with increased risk of overweight or obesity, and predisposition to increased subsequent risk of diabetes. 
Acknowledgements We thank all the participants and the entire EPICNorfolk team. The EPIC-Norfolk cohort is supported by a grant funding from Cancer Research UK and the Medical Research Council, with additional support from the Stroke Association, British Heart Foundation, Research into Ageing, Department of Health and the Wellcome Trust.

Duality of interest The authors declare that there is no duality of interest associated with this manuscript.

\section{References}

1. Wild S, Roglic G, Green A, Sicree R, King H (2004) Global prevalence of diabetes - estimates for the year 2000 and projections for 2030. Diabetes Care 27:1047-1053

2. Wareham NJ, Forouhi NG (2005) Is there really an epidemic of diabetes? Diabetologia 48:1454-1455

3. Narayan KMV, Boyle JP, Thompson TJ, Gregg EW, Williamson DF (2007) Effect of BMI on lifetime risk for diabetes in the U.S. Diabetes Care 30:1562-1566

4. Must A, Spadano J, Coakley EH, Field AE, Colditz G, Dietz WH (1999) The disease burden associated with overweight and obesity. JAMA 282:1523-1529

5. Monteiro PO, Victora CG (2005) Rapid growth in infancy and childhood and obesity in later life - a systematic review. Obes Rev 6:143-154

6. Herman-Giddens ME, Kaplowitz PB, Wasserman R (2004) Navigating the recent articles on girls' puberty in pediatrics: What do we know and where do we go from here? Pediatrics 113:911-917

7. Styne DM (2004) Puberty, obesity and ethnicity. Trends Endocrinol Metab 15:472-478

8. Lee JM, Appugliese D, Kaciroti N, Corwyn RF, Bradley RH, Lumeng JC (2007) Weight status in young girls and the onset of puberty. Pediatrics 119:E624-E630

9. Himes JH, Obarzanek E, Baranowski T, Wilson DM, Rochon J, McClanahan BS (2004) Early sexual maturation, body composition, and obesity in African-American girls. Obesity Res 12:64S$72 \mathrm{~S}$

10. Ong KK, Northstone K, Wells JCK (2007) Earlier mother's age at menarche predicts rapid infancy growth and childhood obesity. PLoS Med 4:737-742

11. Saquib N, Kritz-Silverstein D, Barrett-Connor E (2005) Age at menarche, abnormal glucose tolerance and type 2 diabetes mellitus: The Rancho Bernardo Study. Climacteric 8:76-82

12. Cooper GS, Ephross SA, Sandler DP (2000) Menstrual patterns and risk of adult-onset diabetes mellitus. J Clin Epidemiol 53: $1170-1173$

13. Gambineri A, Pelusi C, Manicardi E et al (2004) Glucose intolerance in a large cohort of Mediterranean women with polycystic ovary syndrome - phenotype and associated factors. Diabetes 53:23532358

14. Day N, Oakes S, Luben R et al (1999) EPIC-Norfolk: study design and characteristics of the cohort. Br J Cancer 80:95-103

15. Wareham NJ, Jakes RW, Rennie KL et al (2003) Validity and repeatability of a simple index derived from the short physical activity questionnaire used in the European Prospective Investigation into Cancer and Nutrition (EPIC) study. Public Health Nutr $6: 407-413$

16. Onland-Moret NC, Peeters PHM, van Gils CH et al (2005) Age at menarche in relation to adult height: the EPIC Study. Am J Epidemiol 162:623-632

17. Must A, Phillips SM, Naumova EN et al (2002) Recall of early menstrual history and menarcheal body size: after 30 years, how well do women remember? Am J Epidemiol 155:672-679

18. Casey VA, Dwyer JT, Coleman KA, Krall EA, Gardner J, Valadian I (1991) Accuracy of recall by middle-aged participants in a longitudinal-study of their body size and indexes of maturation earlier in life. Ann Hum Biol 18:155-166

19. Strotmeyer ES, Steenkiste AR, Foley TP, Berga SL, Dorman JS (2003) Menstrual cycle differences between women with type 1 diabetes and women without diabetes. Diabetes Care 26:1016-1021

20. Freedman D, Khan L, Serdula M, Dietz W, Srinivasan S, Berenson G (2003) The relation of menarcheal age to obesity in childhood and adulthood: the Bogalusa heart study. BMC Pediatrics 3:3

21. Demerath EW, Li JR, Sun SMS et al (2004) Fifty-year trends in serial body mass index during adolescence in girls: the Fels Longitudinal Study. Am J Clin Nutr 80:441-446

22. Kaplowitz PB, Slora EJ, Wasserman RC, Pedlow SE, HermanGiddens ME (2001) Earlier onset of puberty in girls: relation to increased body mass index and race. Pediatrics 108:347-353

23. Ekelund U, Ong KK, Linne Y et al (2007) Association of weight gain in infancy and early childhood with metabolic risk in young adults. J Clin Endocrinol Metab 92:98-103

24. Davison KK, Susman EJ, Birch LL (2003) Percent body fat at age 5 predicts earlier pubertal development among girls at age 9 . Pediatrics 111:815-821

25. Freedman DS, Khan LK, Serdula MK, Dietz WH, Srinivasan SR, Berenson GS (2005) The relation of childhood BMI to adult adiposity: The Bogalusa Heart Study. Pediatrics 115:22-27

26. Silva ID, De Stavola BL, Mann V, Kuh D, Hardy R, Wadsworth MEJ (2002) Prenatal factors, childhood growth trajectories and age at menarche. Int J Epidemiol 31:405-412

27. Fisher D, Baird J, Payne L et al (2006) Are infant size and growth related to burden of disease in adulthood? A systematic review of literature. Int J Epidemiol 35:1196-1210

28. Berkey CS, Frazier AL, Gardner JD, Colditz GA (1999) Adolescence and breast carcinoma risk. Cancer 85:2400-2409

29. Hsieh CC, Trichopoulos D, Katsouyanni K, Yuasa S (1990) Age at menarche, age at menopause, height and obesity as risk-factors for breast-cancer-associations and interactions in an international case-control study. Int J Cancer 46:796-800

30. Lipworth L (1995) Epidemiology of breast-cancer. Eur J Cancer Prev 4:7-30

31. Kelsey JL, Gammon MD, John EM (1993) Reproductive factors and breast-cancer. Epidemiol Rev 15:36-47

32. Cooper GS, Ephross SA, Weinberg CR, Baird DD, Whelan EA, Sandler DP (1999) Menstrual and reproductive risk factors for ischemic heart disease. Epidemiology 10:255-259

33. Ong KK, Ahmed ML, Dunger DB (2006) Lessons from large population studies on timing and tempo of puberty (secular trends and relation to body size): the European trend. Mol Cell Endocrinol 254:8-12 